\title{
Observing a Schwarzschild black hole with finite precision
}

\author{
F. Debbasch ${ }^{1}$ and Y. Ollivier ${ }^{2}$ \\ 1 ERGA, UMR 8112, 4 Place Jussieu, 75231 Paris Cedex 05, France \\ 2 CNRS, UMPA, École normale supérieure de Lyon, 69364 Lyon Cedex 07, France
}

Received 6 May 2004 / Accepted 23 November 2004

\begin{abstract}
We investigate how the space-time of a vacuum Schwarzschild black hole would appear if observed with a finite precision in the measurements of the spatial Kerr-Schild coordinates. For this we use the general procedure for evaluating mean gravitational fields recently presented in Debbasch (2004, Eur. Phys. J. B, 37, 257). It is found that the black hole would then appear as surrounded by an apparent matter characterized by a negative energy density and two different pressures, a negative and a positive one. The total combined effect of the apparent matter leads to a space-time of negative scalar curvature, unlike de Sitter space-time. However, the "magnitude" of the trace-free Ricci tensor does not vanish for this space-time, whereas it does for de Sitter space-time. Possible cosmological implications, concerning the evaluation of the mean density of the Universe and the cosmological constant, are also discussed.
\end{abstract}

Key words. black hole physics - relativity - cosmology: theory - gravitation - cosmological parameters

\section{Notation}

In this article, space-time indices running from 0 to 3 will be indicated by Greek letters. The metric signature will be $(+,-,-,-)$. We also have chosen, as a rule, not to use the socalled intrinsic notation in differential geometry, but to use the notation standard in physics, which denotes each tensor by its components.

\section{Introduction}

Every observation is necessarily finite i.e. it deals with a finite number of quantities, observed or measured with a finite precision. This explains why mean field theories play such an important role in physics. It will therefore come as no surprise that developing a mean field approach to relativistic gravitation has been the subject of active research for more than a decade (Futamase 1991, 1993; Kasai 1992; Futamase 1996; Zalaletdinov 1997; Buchert 2000, 2001). This conceptually and practically crucial problem has been recently solved in a rather general way (Debbasch 2004, 2005). It has been shown that, given a statistical ensemble $\Sigma$ of space-times sharing a common topology, it makes both mathematical and physical sense to define the mean (or apparent, or coarse-grained) space-time associated with this ensemble as a space-time of the same topology, but where the gravitational field is represented by a metric which is simply the average of the metrics corresponding to the various space-time members of $\Sigma$.

This apparently very innocuous result has however several exotic consequences. One of them is that the separation between the gravitational field and the matter degrees of freedom actually depends on the precision of the observations
(Debbasch 2004). Let us consider the following particular situation. Suppose a region $\mathcal{D}$ of space-time is observed with a certain finite precision and that the observations indicate that no matter is present in $\mathcal{D}$, but only a non-vanishing gravitational field. Then, generically, other observations carried out with a different (greater or lesser) precision will indicate that $\mathcal{D}$ contains both matter and a non-vanishing gravitational field. The aim of this article is to investigate this "purely relativistic" effect on a perhaps academic but de facto simple and hopefully illuminating example, where most calculations can be made completely explicit. More precisely, we consider the Schwarzschild black hole, which is one of the simplest vacuum solutions to Einstein's equation and we study how a finite precision in coordinate measurements can make it look like a non-vacuum solution to the general relativistic field equations.

The material is organized as follows. Section 2 reviews some basic results about ensembles of space-times and about the properties of the mean or coarse-grained gravitational field with which they are associated. Section 3 introduces the particular statistical ensemble which will be considered in this article; it is notably explained why averaging over this statistical ensemble can be interpreted as observing a Schwarzschild black hole with a finite precision. In Sect. 4, we calculate the mean metric associated with this statistical ensemble and, in Sect. 5, the stress-energy tensor of the apparent matter which seems to surround the black hole is explicitly evaluated as a function of the coarse-graining; the calculation is a perturbative one and is valid for points whose radial (Schwarzschild) coordinates are much larger than the coarse-graining itself. At lowest order, it is found that the apparent matter can be characterized by an energy density and two different pressures; the energy density and one of the pressures is negative, while the 
other pressure is positive. All three quantities decrease towards zero as the radial coordinates tends to infinity. We also show that the total effect of this apparent matter is to induce a negative scalar curvature in space-time. Thus, by coarse-graining, the vacuum surrounding the Schwarzschild black hole acquires a stress-energy tensor which generates a space-time of negative curvature. This obviously brings to mind de Sitter space-time, the negative curvature of which is generated by a non-vanishing positive cosmological constant. Section 6 provides an in-depth discussion of the results presented in this article, including possible cosmological implications. In particular, the similarities and differences between the apparent vacuum stress-energy due to the coarse graining and the stress-energy corresponding to a cosmological constant are analyzed. As a conclusion, we provide a summary of the new material and we also mention and discuss briefly some of the many possible extensions of this work, including several more realistic situations of direct astrophysical and/or cosmological relevance.

\section{Mean gravitational fields}

\subsection{Ensembles of space-times}

Let us consider a statistical ensemble $\Sigma$ of space-times $\mathcal{M}(\omega)$, $\omega \in \Omega$. $\Omega$ is an arbitrary probability space (Grimmett \& Stirzaker 1994); each member of the ensemble $\Sigma$ is a differentiable manifold endowed with a metric $g(\omega)$, the Levi-Civita connection $\Gamma(\omega)$ associated with $g(\omega)$ (Nakahara 1990) and a stress-energy tensor $T(\omega)$.

We will restrict the discussion by supposing that all space-times in our statistical ensemble share the same topology and are distinguished only by their respective gravitational fields. More precisely, we suppose that there is a single manifold $M$ underlying all of our space-times $\mathcal{M}(\omega)$ (such an $M$ represents the set of points of space-time), so that $\mathcal{M}(\omega)$ is $M$ equipped with an $\omega$-dependent metric field $g(\omega)$. One can thus choose an atlas common to all space-times, so that for any chart (i.e. any local coordinate system $(x)), \mathcal{M}(\omega)$ is represented by an $\omega$-dependent metric field $g_{\mu \nu}(x, \omega)$.

Each space-time $\mathcal{M}(\omega)$ verifies the Einstein equation (Wald 1984). One thus has:

$\mathcal{E}_{\mu v}(g(\omega)) \equiv R_{\mu v}(\omega)-\frac{1}{2} R(\omega) g_{\mu \nu}(\omega)=\chi g_{\mu \alpha}(\omega) g_{v \beta}(\omega) T^{\alpha \beta}(\omega),(1)$

where the $R_{\mu \nu}$ 's are the coordinate-basis components of the Ricci tensor, $R$ is the trace of this tensor and $\chi$ is the gravitational constant. The combination on the left-hand side of (1) is usually called the Einstein tensor, hence the notation. Unless otherwise specified, the units used in the rest of this article are so chosen that $\chi=8 \pi$ (Wald 1984).

\subsection{Definition of a mean space-time}

It has been shown in Debbasch (2004) that the statistical ensemble $\Sigma$ of space-times can be used to define a single, mean Einstein space-time $\bar{M}$ and that, by construction, the atlas common to all members of $\Sigma$ can be used as an atlas for $\overline{\mathcal{M}}$. $\overline{\mathcal{M}}$ is endowed with a metric $\bar{g}$ which is the average of the metrics $g(\omega)$ over $\omega$; one thus has, for all $x$ :

$\bar{g}(x)=\langle g(x, \omega)\rangle$,

where the brackets on the right-hand side indicate an average over the statistical ensemble $\Sigma$.

The connection of the mean space-time $\bar{M}$ is simply the Levi-Civita connection associated with the metric $\bar{g}$ and will be conveniently called the mean connection. Since the relations linking the components $g_{\mu \nu}$ of an arbitrary metric $g$ to the Christoffel symbols $\Gamma_{\mu \nu}^{\alpha}$ of its Levi-Civita connection are non-linear, the Christoffel symbols of the mean connection are not identical to the averages of the Christoffel symbols associated with the various space-times $\mathcal{M}(\omega)$. Note however that the so-called "covariant" connection coefficients $\Gamma_{\mu, \alpha \beta}(\omega) \equiv g_{\mu v}(\omega) \Gamma_{\alpha \beta}^{\nu}(\omega)$ depend linearly on the metric components $g_{\mu v}(\omega)$, so that $\bar{\Gamma}_{\mu, \alpha \beta}=\left\langle\Gamma_{\mu, \alpha \beta}(\omega)\right\rangle$. This point is thoroughly elaborated upon in Debbasch (2004), where a complete discussion of the mathematical and physical motivations for definition (2) can also be found.

Because the Einstein tensor depends non-linearly on the metric, the Einstein tensor $\overline{\mathcal{E}}=\mathcal{E}(\bar{g})$ associated with the mean metric does not generally coincide with the average of the Einstein tensors $\mathcal{E}(g(\omega))$. The tensor $\overline{\mathcal{E}}$ is nevertheless the Einstein tensor of the mean space-time. It therefore defines, via the Einstein equation, a stress-energy tensor $\bar{T}$ for the mean space-time:

$\mathcal{E}_{\mu \nu}(\bar{g})=\chi \bar{g}_{\mu \alpha} \bar{g}_{v \beta} \bar{T}^{\alpha \beta}$.

Since $\mathcal{E}_{\mu v}(\bar{g}) \neq\left\langle\mathcal{E}_{\mu v}(g(\omega))\right\rangle$, the mean stress-energy tensor $\bar{T}^{\alpha \beta}$ is generally different from the average $\left\langle T^{\alpha \beta}(\omega)\right\rangle$ of the stress-energy tensors of the space-times in the statistical distribution. It is therefore convenient to introduce the generally non-vanishing tensor field $\Delta T$, defined on $\overline{\mathcal{M}}$ by:

$\Delta T^{\alpha \beta}=\bar{T}^{\alpha \beta}-\left\langle T^{\alpha \beta}(\omega)\right\rangle$.

This difference $\Delta T$ can be interpreted as the stress-energy tensor of an "apparent matter" which contributes, along with the average $\langle T(\omega)\rangle$ of the stress-energy associated with the "real" matter present in the various original space-times $\mathcal{M}(\omega)$, to creating the mean gravitationnal field $\bar{g}$ :

$\mathcal{E}_{\mu \nu}(\bar{g})=\chi \bar{g}_{\mu \alpha} \bar{g}_{v \beta}\left(\left\langle T^{\alpha \beta}(\omega)\right\rangle+\Delta T^{\alpha \beta}\right)$.

In particular, the vanishing of $T(\omega)$ for all $\omega$ does not necessarily imply the vanishing of $\bar{T}$. The mean stress-energy tensor $\bar{T}$ can therefore be non-vanishing in regions where the unaveraged stress-energy tensor actually vanishes. A general dicussion of this and other perhaps unexpected consequences of definition (2) can be found in Debbasch (2004, 2005). The particular cases when the matter is made of an electromagnetic field and/or of a possibly charged perfect fluid is also addressed in depth by Debbasch (2004).

The goal of this article is to present a simple case when $T(\omega)$ vanishes for all $\omega$ and $\Delta T$ is nevertheless non-zero. 


\section{An ensemble of space-times representing a Schwarzschild black hole observed with a finite precision}

The so-called Kerr-Schild form (Chandrasekhar 1992; Kramer et al. 1980) of the (vacuum) Schwarzschild metric is:

$\mathrm{d} s^{2}=\mathrm{d} t^{2}-\mathrm{d} \boldsymbol{r}^{2}-\frac{2 M}{r}\left(\mathrm{~d} t+\frac{r}{r} \cdot \mathrm{d} \boldsymbol{r}\right)^{2}$.

The parameter $M$ represents the mass of the black hole and $\boldsymbol{r}$ stands for the set of three "spatial" coordinates $x, y, z$. We have also retained the standard and natural notations:

$\mathrm{d} \boldsymbol{r}^{2}=\mathrm{d} x^{2}+\mathrm{d} y^{2}+\mathrm{d} z^{2}$

and

$\boldsymbol{r} \cdot \mathrm{d} \boldsymbol{r}=x \mathrm{~d} x+y \mathrm{~d} y+z \mathrm{~d} z$.

The Kerr-Schild coordinates (as opposed to the perhaps more standard Schwarzschild coordinates (Wald 1984)) are particularly natural and convenient because they form a single-chart atlas of the whole Schwarzschild space-time (Chandrasekhar 1992), the only singularities of this space-time being the points on the "line" $x=y=z=0$, where the components of the metric tensor (6) are themselves singular.

Let us now introduce an at this stage arbitrary $\omega$ in $\mathbb{R}^{3}$ and consider the $\omega$-dependent metric

$\mathrm{d} s_{\omega}^{2}=\mathrm{d} t^{2}-\mathrm{d} \boldsymbol{r}^{2}-\frac{2 M}{\rho}\left(\mathrm{d} t+\frac{\boldsymbol{r}-\boldsymbol{\omega}}{\rho} \cdot \mathrm{d} \boldsymbol{r}\right)^{2}$,

where

$\rho^{2}=(\boldsymbol{r}-\boldsymbol{\omega})^{2}=r^{2}+\omega^{2}-2 \boldsymbol{r} \cdot \boldsymbol{\omega}$.

Note that the "original" Schwarzschild space-time associated with (6) is actually $\mathcal{M}(0)$; also observe that, for any $(t, r)$ and $\omega$ :

$g(t, \boldsymbol{r}, \omega)=g(t, \boldsymbol{r}-\omega, 0)$,

so that $g(t, r, \omega)$ represents an ordinary black hole centered around point $\omega$.

Let $\Omega=\left\{\omega \in \mathbb{R}^{3} ; \omega^{2} \leqslant a^{2}\right\}$ where $a$ is a fixed, positive real constant; $\Omega$ is the usual 3-ball of radius $a$ in Euclidean space $\mathbb{R}^{3}$. We will use as volume measure on $\Omega$ the usual (Lebesgue) measure $\mathrm{d}^{3} \omega$ and, with this measure, the total volume of $\Omega$ is simply $V_{a}=4 \pi a^{3} / 3$. The measure $\mathrm{d}^{3} \omega$ thus defines a probability measure on $\Omega$ by:

$p(\omega) \mathrm{d}^{3} \omega=\frac{1}{V_{a}} \mathrm{~d}^{3} \omega$.

We now define a statistical ensemble $\Sigma$ of space-times by $\Sigma=$ $\{\mathcal{M}(\omega) ; \omega \in \Omega\}$ and use on $\Sigma$ the probability measure (12).

The remainder of this article is devoted to investigating some properties of the average space-time $\overline{\mathcal{M}}$ which can be constructed out of this ensemble by the procedure outlined in the previous section. Before embarking on any calculation, let us give a physical motivation for considering the ensemble $\Sigma$.

At any point $(t, r)$ in space-time, the value $\bar{g}(t, \boldsymbol{r})$ taken by the metric $\bar{g}$ of the average space-time $\overline{\mathcal{M}}$ is simply the average of $g(t, \boldsymbol{r}, \boldsymbol{\omega})$ over $\boldsymbol{\omega}$. One thus has, by Eq. (11):

$\bar{g}(t, \boldsymbol{r})=\langle g(t, \boldsymbol{r}-\omega, 0)\rangle$.
This shows that, at any given point $(t, r)$ in space-time, the metric $\bar{g}$ is simply the average of the original metric (6) over the 3 -ball of radius $a$ centered at $(t, r)$.

The metric $\bar{g}$ can therefore be interpreted as the original metric $g(0)$ observed, in the chosen coordinates, with the finite "spatial" resolution $a$. It thus represents a Schwarzschild black hole observed with a finite precision. Indeed, suppose that, by some observational procedure, we can have experimental access to the metric tensor field $g$ but suppose also that the determination of each "spatial" Kerr-Schild coordinate is subject to an error of order $a$. Then, instead of measuring, say $g(t, \boldsymbol{r})$, we actually measure $g(t, \boldsymbol{r}-\omega)$ for some randomly chosen $\omega$ of norm at most $a$ (in the sense of Eq. (7)). The "observed" or "measured" metric will then precisely be $\bar{g}(t, \boldsymbol{r})=\langle g(t, \boldsymbol{r}-\omega, 0)\rangle$.

As explained in the previous section, the average metric $\bar{g}$ defines by Einstein's equation an a priori non-vanishing stress-energy tensor $\bar{T}$. In other words, although each metric $g(t, r, \omega)$ in the ensemble $\Sigma$ is a solution of Einstein's equation in vacuum, the average metric $\bar{g}$ is not. If measurements are made with a finite "spatial" resolution $a$, the observed metric $\bar{g}$ can only be consistently understood as a solution of Einstein's equation if one takes into account an "apparent" matter caracterized by the stress-energy tensor $\bar{T}$. We now want to investigate the properties of this matter in greater detail.

\section{Determination of the mean metric}

\subsection{Kerr-Schild coordinates}

We first begin by determining the average metric $\bar{g}$, fully defined by $\bar{g}(t, \boldsymbol{r})=\langle g(t, \boldsymbol{r}-\omega, 0)\rangle$. For obvious physical reasons, one is only interested in evaluating the mean metric $\bar{g}$ at points $(t, r)$ for which $r \gg a$. This we will now do, pushing all expansions at order two in $a / r$.

Equation (9) can be rewritten as:

$$
\begin{aligned}
\mathrm{d} s^{2}= & \mathrm{d} t^{2}-\mathrm{d} \boldsymbol{r}^{2}-\frac{2 M}{\rho} \\
& \times\left(\mathrm{d} t^{2}+\frac{2 \mathrm{~d} t}{\rho} \mathrm{d} \boldsymbol{r} \cdot(\boldsymbol{r}-\boldsymbol{\omega})+\frac{1}{\rho^{2}}(\mathrm{~d} \boldsymbol{r} \cdot(\boldsymbol{r}-\boldsymbol{\omega}))^{2}\right)
\end{aligned}
$$

where as above, $\rho^{2}=(\boldsymbol{r}-\boldsymbol{\omega})^{2}$.

To proceed, one needs to expand the various powers of $1 / \rho$ which enter (14) into powers of $\boldsymbol{r}$ and $\omega / \boldsymbol{r}$.

The powers of $1 / \rho$. Here we begin to use the assumption that $r \gg a$. All subsequents expansions are at order 2 in $\omega / r$.

Expanding $1 / \rho=1 / \sqrt{r^{2}+\omega^{2}-2 \boldsymbol{r} \cdot \omega}$ at order 2 in $\omega / r$ we get

$\frac{1}{\rho}=\frac{1}{r}\left(1+\frac{\boldsymbol{r} \cdot \omega}{r^{2}}-\frac{1}{2} \frac{\omega^{2}}{r^{2}}+\frac{3}{2} \frac{(\boldsymbol{r} \cdot \omega)^{2}}{r^{4}}\right)$

$\frac{1}{\rho^{2}}=\frac{1}{r^{2}}\left(1+2 \frac{\boldsymbol{r} \cdot \boldsymbol{\omega}}{r^{2}}-\frac{\omega^{2}}{r^{2}}+4 \frac{(\boldsymbol{r} \cdot \boldsymbol{\omega})^{2}}{r^{4}}\right)$

$\frac{1}{\rho^{3}}=\frac{1}{r^{3}}\left(1+3 \frac{\boldsymbol{r} \cdot \omega}{r^{2}}-\frac{3}{2} \frac{\omega^{2}}{r^{2}}+\frac{15}{2} \frac{(\boldsymbol{r} \cdot \omega)^{2}}{r^{4}}\right)$. 
Intermediate forms of the mean metric. We now plug these expansions into Eq. (14) and average for $\omega$ in the ball $\Omega$ of radius $a$. By symmetry, it is clear that the average of $\omega$ is 0 , as well as the average of all terms containing an odd power of $\omega$. We get

$$
\begin{aligned}
& \left\langle\mathrm{d} s^{2}\right\rangle=\mathrm{d} t^{2}-\mathrm{d} \boldsymbol{r}^{2}-\frac{2 M}{r} \mathrm{~d} t^{2}\left(1-\frac{1}{2 r^{2}}\left\langle\omega^{2}\right\rangle+\frac{3}{2 r^{4}}\left\langle(\boldsymbol{r} \cdot \omega)^{2}\right\rangle\right) \\
& -\frac{4 M}{r^{2}} \mathrm{~d} t \mathrm{~d} \boldsymbol{r} \cdot\left(\boldsymbol{r}\left(1-\frac{1}{r^{2}}\left\langle\omega^{2}\right\rangle+\frac{4}{r^{4}}\left\langle(\boldsymbol{r} \cdot \omega)^{2}\right\rangle\right)-\frac{2}{r^{2}}\langle\omega(\boldsymbol{r} \cdot \omega)\rangle\right) \\
& -\frac{2 M}{r^{3}}\left(\begin{array}{l}
\left\langle(\mathrm{d} \boldsymbol{r} \cdot(\boldsymbol{r}-\omega))^{2}\right\rangle+\frac{3}{r^{2}}\left\langle(\boldsymbol{r} \cdot \omega)(\mathrm{d} \boldsymbol{r} \cdot(\boldsymbol{r}-\omega))^{2}\right\rangle \\
\left.-\frac{3}{2 r^{2}}\left\langle\omega^{2}(\mathrm{~d} \boldsymbol{r} \cdot(\boldsymbol{r}-\omega))^{2}\right\rangle+\frac{15}{2 r^{4}}\left\langle(\mathrm{~d} \boldsymbol{r} \cdot(\boldsymbol{r}-\omega))^{2}(\boldsymbol{r} \cdot \omega)^{2}\right\rangle\right)
\end{array}\right.
\end{aligned}
$$

We thus need to compute the averages of several functions of $\boldsymbol{r}$ and $\omega$. Symmetry arguments make the task easier. Remember that the average is taken on the Euclidean 3-ball of radius $a$. Since $a$ is supposed to be much smaller than $r$, we only keep order-2 terms in $a$.

$$
\begin{aligned}
& \left\langle\omega^{2}\right\rangle=\frac{3 a^{2}}{5} ;\left\langle(\boldsymbol{r} \cdot \omega)^{2}\right\rangle=\frac{a^{2} r^{2}}{5} ;\langle\omega(\boldsymbol{r} \cdot \omega)\rangle=\frac{a^{2}}{5} \boldsymbol{r} \\
& \left\langle(\mathrm{d} \boldsymbol{r} \cdot(\boldsymbol{r}-\omega))^{2}\right\rangle=r^{2} \mathrm{~d} r^{2}+\frac{a^{2}}{5} \mathrm{~d} \boldsymbol{r}^{2} \\
& \left\langle(\boldsymbol{r} \cdot \omega)(\mathrm{d} \boldsymbol{r} \cdot(\boldsymbol{r}-\omega))^{2}\right\rangle=-\frac{2 a^{2} r^{2}}{5} \mathrm{~d} r^{2} \\
& \left\langle\omega^{2}(\mathrm{~d} \boldsymbol{r} \cdot(\boldsymbol{r}-\omega))^{2}\right\rangle=\frac{3 a^{2} r^{2}}{5} \mathrm{~d} r^{2}+\text { higher-order terms } \\
& \left\langle(\boldsymbol{r} \cdot \omega)^{2}(\mathrm{~d} \boldsymbol{r} \cdot(\boldsymbol{r}-\omega))^{2}\right\rangle=\frac{a^{2} r^{4}}{5} \mathrm{~d} r^{2}+\text { higher-order terms. }
\end{aligned}
$$

Plugging this into expression (18) for $\left\langle\mathrm{d} s^{2}\right\rangle$ we get the somewhat simpler form

$$
\begin{aligned}
\left\langle\mathrm{d} s^{2}\right\rangle= & \mathrm{d} t^{2}-\mathrm{d} r^{2}\left(1+\frac{2 M a^{2}}{5 r^{3}}\right)-\frac{2 M}{r} \mathrm{~d} t^{2} \\
& -\frac{4 M}{r} \mathrm{~d} t \mathrm{~d} r\left(1-\frac{a^{2}}{5 r^{2}}\right)-\frac{2 M}{r} \mathrm{~d} r^{2}\left(1-\frac{3 a^{2}}{5 r^{2}}\right)
\end{aligned}
$$

which is the expression of the mean metric for a Schwarzschild black hole observed, in the retained coordinate system, with finite "spatial" resolution $a$. Of course $a=0$ gives back the usual metric (6). The deformation is of second order in $a$ due to the symmetry of the ensemble $\Sigma$.

Comparing Eqs. (24) to (6), we see that the role played by $\mathrm{d} \boldsymbol{r}^{2}$ in (6) is now played by $\mathrm{d} \boldsymbol{r}^{2}\left(1+\frac{2 M a^{2}}{5 r^{3}}\right)$. This suggests the introduction of the new "spatial" coordinates $\boldsymbol{R}$, defined by:

$$
\boldsymbol{R}=\boldsymbol{r}\left(1+\frac{M a^{2}}{5 r^{3}}\right)
$$

observe that $\boldsymbol{R}$ is equivalent to $\boldsymbol{r}$ at infinity.

Expressing $\left\langle\mathrm{d} s^{2}\right\rangle$ in terms of the coordinates $(t, \boldsymbol{R})$ yields:

$$
\begin{aligned}
& \left\langle\mathrm{d} s^{2}\right\rangle=\mathrm{d} t^{2}-\mathrm{d} \boldsymbol{R}^{2}-\frac{2 M}{R} \mathrm{~d} t^{2}\left(1+\frac{M a^{2}}{5 R^{3}}\right) \\
& -\frac{4 M}{R} \mathrm{~d} t \mathrm{~d} R\left(1+\left(\frac{3 M}{R}-1\right) \frac{a^{2}}{5 R^{2}}\right)-\frac{2 M}{R} \mathrm{~d} R^{2}\left(1+\frac{M a^{2}}{R^{3}}\right),
\end{aligned}
$$

or, equivalently,

$$
\begin{aligned}
\left\langle\mathrm{d} s^{2}\right\rangle= & \mathrm{d} t^{2}-\mathrm{d} \boldsymbol{R}^{2}-\frac{2 M}{R}\left(\mathrm{~d} t\left(1+\frac{M a^{2}}{10 R^{3}}\right)\right. \\
& \left.+\mathrm{d} R\left(1+\frac{M a^{2}}{2 R^{3}}\right)\right)^{2}+\frac{4 M a^{2}}{5 R^{3}} \mathrm{~d} t \mathrm{~d} R .
\end{aligned}
$$

Both above expressions are correct at order 2 in $a / R$. They represent the finite-resolution version of Eq. (6). By a slight extension of the common terminology, we will say that the coordinates $(t, \boldsymbol{R})$ are Kerr-Schild coordinates for the average space-time $\overline{\mathcal{M}}$. Formally speaking, the only singularities of the metric (26) are the points on the line $R=0$. Thus, the Kerr-Schild coordinates form a single-chart atlas of the spacetime equipped with metric (26). Note however that Eq. (26) was derived from Eq. (6) under the assumption that $r \gg a$, which implies $R \gg a$ via Eq. (25). The singularity of Eq. (26) at $R=0$ is therefore not "physical", i.e. it does not entail that the mean space-time $\overline{\mathcal{M}}$ is singular at $R=0$. Moreover, the very notion of a mean space-time probably makes no physical sense for values of $R$ comparable or inferior to the coarse graining parameter $a^{1}$.

\subsection{Schwarzschild coordinates}

By suitably choosing a new time-variable $\tau(t, r)$, the metric (6) can be put into the well-known form (Wald 1984; Chandrasekhar 1992):

$\mathrm{d} s^{2}=\left(1-\frac{2 M}{r}\right) \mathrm{d} \tau^{2}-\frac{1}{1-2 M / r} \mathrm{~d} r^{2}-r^{2} \mathrm{~d} \Gamma^{2}$,

where $\mathrm{d} \Gamma$ stands for the elementary solid angle associated with the three "spatial" coordinates $\boldsymbol{r}$. The coordinates $(\tau, \boldsymbol{r})$ are called the Schwarzschild coordinates. As already mentioned, these coordinates do not constitute a single-chart atlas of the Schwarzschild space-time. They are however relevant for observers outside the black hole and, if only because no term in $\mathrm{d} r \mathrm{~d} \tau$ appears in Eq. (28), they also present undeniable computational advantages. It is therefore natural to seek a new coordinate system which would play for the mean metric (26) the role the usual Schwarzschild coordinate system plays for vacuum black holes. By extension, these new coordinates will be called the Schwarzschild coordinates of the mean metric.

They can be obtained by keeping $\boldsymbol{R}$ as "spatial" coordinates and by merely introducing a new time-coordinate $T$, defined in terms of $t$ and $\boldsymbol{R}$ by a relation of the form:

$\mathrm{d} t=\mathrm{d} T+\alpha(R) \mathrm{d} R$.

In fact it is not even necessary to compute $\alpha(R)$ explicitly to obtain the expression of the mean metric in Schwarzschild coordinates: indeed, the transformation (29) does not change the determinant of the metric (since this transformation is of determinant 1), and it does not change the term in front of $\mathrm{d} T^{2}$ either. A simple computation shows that the determinant of the

\footnotetext{
1 Just as it makes no physical sense, for example, to speak of the electric field created by an electrostatic dipole at distances comparable or inferior to the caracteristic spatial extension of the charge distribution modelled by the dipole.
} 
$(t, R)$-part of the metric (26) is -1 at order 2 in $a / R$. So the final metric will be of determinant -1 and, therefore, the terms in front of $\mathrm{d} T^{2}$ and $\mathrm{d} R^{2}$ will be the inverse of each other. Since the $g_{T T}$ component is known from Eq. (26), so is the $g_{R R}$ component. Naturally, this simple reasoning can be double-checked through a straightforward but rather long direct computation of $\alpha(R)$. Indeed, the choice:

$\alpha(R)=\frac{2 M}{R} \frac{1}{1-2 M / R}\left(1-\frac{a^{2}}{5 R^{2}} \frac{1}{1-2 M / R}\left(\frac{4 M^{2}}{R^{2}}-\frac{5 M}{R}+1\right)\right)$

ensures the vanishing of the mixed metric component $g_{T R}$ and the $g_{R R}$ component of the metric in Schwarzschild coordinates can then be obtained by direct computation.

The final form of the mean metric in Schwarzschild coordinates is therefore (with $\mathrm{d} \Gamma$ the usual Euclidean solid angle element):

$\left\langle\mathrm{d} s^{2}\right\rangle=F(R) \mathrm{d} T^{2}-G(R) \mathrm{d} R^{2}-R^{2} \mathrm{~d} \Gamma^{2}$

where

$F(R)=1-\frac{2 M}{R}-\frac{2 a^{2} M^{2}}{5 R^{4}}$

and

$G(R)=\frac{1}{F(R)}$

or, when $R-2 M \gg a^{2} M^{2} / R^{3}$,

$G(R)=\frac{1}{1-2 M / R}+\frac{2 a^{2} M^{2}}{5 R^{4}}\left(\frac{1}{1-2 M / R}\right)^{2}$.

There are a few simple but important remarks to be made about this result.

1. Expression (30) shows that the Schwarzschild coordinates cannot be used in the whole space-time $\bar{M}$ but are only valid in the domains $R>2 M$ and $R<2 M$. On the other hand, the Kerr-Schild coordinates do form a single-chart atlas of $\overline{\mathcal{M}}$.

2. Of course, when $a=0$, the preceding expression reduces to the metric (28) of a vaccum, non-rotating black hole in Schwarzschild coordinates. The average space-time is also static (Wald 1984), as can be seen from the absence of $\mathrm{d} T \mathrm{~d} R$ term in Eq. (31). This was predictable since the mean space-time results from averaging static space-times.

3. The deformation of Eq. (31) with respect to Eq. (28) is of second order in $a / R$, due to symmetry of our statistical ensemble.

4. As noted above, the determinant of the $(T, R)$-part of the metric is -1 :

$F(R)=1 / G(R)$

at order 2 in $a / R$. This property of the mean metric is shared by the metrics of the unaveraged space-times in Schwarzschild coordinates.

5. The only assumption that was made is that the coarsegraining $a$ is much smaller than $R$. In particular, we have not assumed that $R$ is large compared to the Schwarzschild radius $M$ of the black hole: if the uncertainty $a$ is small compared to $M$, then our estimate is valid even for $R \approx 2 M$ i.e., very close to the horizon of the unaveraged Schwarzschild black hole.
6. The metric components in the coordinate basis associated with $T, R, \theta$ and $\phi$ exhibit singularities for two different values of $R$. The singularity at $R=0$ also appears in the form (27) of the average metric (see discussion above). As in the Schwarzschild case, this is a real singularity of the geometry defined by the metric (31); but let us stress again that the very notion of a mean space-time probably makes no physical sense for values of $R$ comparable or inferior to the coarse graining parameter $a$. The other singularity of Eq. (31) occurs for $F(R)=0$, namely $5 R^{4}-10 M R^{3}-2 a^{2} M^{2}=0$. Solving this equation at order 2 in $a / M$ (which is the order at which the mean metric has been computed), one finds that this singularity occurs at $R=2 M\left(1+a^{2} / 40 M^{2}\right)$. This is a mere coordinate singularity, the occurence of which parallels the presence of an apparent singularity at $R=2 M$ for the components of Schwarzschild metric (28) in the basis associated with the usual Schwarzschild coordinates. The fact that the singularity at $R=2 M\left(1+a^{2} / 40 M^{2}\right)$ is only due to the choice of coordinates in Eq. (31) can be checked in two different ways. First, this singularity is absent from the metric components (27) in the coordinate basis associatedwith $(t, R, \theta, \phi)$. Second, the curvature tensor associated with Eq. (31) is well behaved everywhere, except at $R=0$. In particular, the scalar curvature $\mathcal{R}$ of the mean space-time will be calculated in the next Section and is given by Eq. (39). It scales as $R^{-6}$ and is everywhere finite, except at $R=0$. The apparent singularity is thus due to the singular behaviour of the coordinate change defined by Eqs. (29) and (30) at $R=2 M\left(1+a^{2} / 40 M^{2}\right)$.

\section{Energy and pressure of apparent matter}

Let us insist that the mean metric (31) is not a solution of Einstein's equation in vacuum. We now wish to evaluate the stress-energy tensor $\bar{T}$ corresponding to this metric. This stress-energy tensor is the one that would be inferred by an observer having access to the coarse-grained metric (31); it therefore constitutes apparent matter that would be "detected" by any observer mapping the gravitational field with the finite spatial precision $a$ (in Kerr-Schild coordinates). We will restrict our discussion by investigating the properties of this apparent matter in the region $R>2 M$ only (the stress-energy tensor $\bar{T}$ in the region $R<2 M$ can be deduced similarly).

The use of Schwarzschild coordinates in the region $R>2 M$ allows for a very easy computation of $\bar{T}$. Indeed, for metrics of the form $e^{v} \mathrm{~d} T^{2}-e^{\lambda} \mathrm{d} R^{2}-R^{2} \mathrm{~d} \Gamma^{2}$ the stress-energy tensor can be readily expressed in terms of $\lambda$ and $v$ (see for example, Landau $\&$ Lifshitz 1975, Eqs. (100,2), (100,4), (100,6), or Wald 1984). In the case at hand, the calculation further simplifies since, first, $\lambda=-v($ since $F=1 / G)$ and, second, all functions are independent of the time coordinate $T$. One thus immediately gets:

$$
\begin{array}{ll}
8 \pi \bar{T}_{0}^{0}=-\frac{6 a^{2} M^{2}}{5 R^{6}} ; & 8 \pi \bar{T}_{1}^{1}=-\frac{6 a^{2} M^{2}}{5 R^{6}} \\
8 \pi \bar{T}_{2}^{2}=\frac{12 a^{2} M^{2}}{5 R^{6}} ; & 8 \pi \bar{T}_{3}^{3}=\frac{12 a^{2} M^{2}}{5 R^{6}}
\end{array}
$$


all other components of $\bar{T}$ vanish, so that the stress-energy tensor $\bar{T}$ is diagonal in Schwarzschild coordinates.

As is well-known (Landau \& Lifshitz 1975; Wald 1984), the component $\bar{T}_{0}^{0}$ can be interpreted as an energy density $\varepsilon$; in the present case, $\varepsilon$ represents the energy-density of the apparent matter and the pressure of this matter in direction $i$ is similarly given by $-T_{i}^{i}$.

We thus have:

$\varepsilon=-\frac{1}{8 \pi} \frac{6 a^{2} M^{2}}{5 R^{6}} ; \quad p_{1}=\frac{1}{8 \pi} \frac{6 a^{2} M^{2}}{5 R^{6}} ;$

$p_{2}=-\frac{1}{8 \pi} \frac{12 a^{2} M^{2}}{5 R^{6}} ; \quad p_{3}=-\frac{1}{8 \pi} \frac{12 a^{2} M^{2}}{5 R^{6}}$.

In particular, the apparent energy density is negative, and the pressure tensor is anisotropic; the radial direction (pointing towards the center of the black hole) is associated with a positive pressure whereas the single pressure associated with both perpendicular directions is negative.

It is also interesting to evaluate the (scalar) curvature $\mathcal{R}$ of the mean space-time; Einstein's Eq. (1) leads directly to:

$R_{\mu}^{\mu}-\frac{1}{2} \mathcal{R} g_{\mu}^{\mu}=-\mathcal{R}=\chi\left(\varepsilon-p_{1}-p_{2}-p_{3}\right)$

with $\chi=8 \pi$ in the chosen units, so that:

$\mathcal{R}=-\frac{12 a^{2} M^{2}}{5 R^{6}}$.

The averaging procedure thus confers on the space-time an apparent, strictly negative scalar curvature. In other words, after coarse-graining, the vacuum of the original Schwarzschild black hole appears endowed with a negative scalar curvature. This striking conclusion cannot but bring to mind the recent observation (Spergel et al. 2003) of a positive, non-vanishing cosmological constant $\Lambda$, which also endows vacuum regions of space-time with a negative scalar curvature (Hawking \& Ellis 1973; Kolb \& Turner 1990; Peebles 1993) $\mathcal{R}_{\Lambda}=-4 \Lambda \times 8 \pi$. This point will be further discussed below.

\section{Discussion}

Physical interpretation, for black holes, of the retained averaging. We wish first to discuss again the physical significance of the statistical ensemble of space-times $\Sigma$ introduced in Sect. 4. As argued in that section, averaging the metric over this statistical ensemble leads to a new, mean metric $\bar{g}$ which represents the gravitationnal field "detected" by someone who observes a vacuum Schwarzschild black hole with a finite precision $a$ in the measurements of the "spatial" Kerr-Schild coordinates. The point we would like to stress here is that the mean metric $\bar{g}$ does not represent the gravitational field detected by someone who observes a Schwarzschild black hole with finite precision $a$ in the measurements of other coordinates, e.g. the "spatial" Schwarzschild coordinates. To obtain the mean metric $\bar{g}^{\prime}$ in that latter case, one would have to start with a new ensemble of space-times $\Sigma^{\prime}$, constructed from Eq. (28) exactly as $\Sigma$ is constructed from Eq. (6), and evaluate $\bar{g}^{\prime}$ as an average over this new ensemble $\Sigma^{\prime 2}$. There is obviously no reason

\footnotetext{
2 Arbitrary finite precisions in the measurements of any coordinates or parameters on which the metric depends can naturally be taken into account in a similar fashion.
}

why the metric $\bar{g}^{\prime}$ should be identical to (28). Indeed, expression (28) represents the metric $\bar{g}$ (not $\bar{g}$ ), but in a coordinate system different from the one used in (26).

As for $\bar{g}^{\prime}$, it can also be expressed in various coordinate systems. In one of them, which one could call the Schwarzschild coordinate system for $\bar{g}^{\prime}$, this metric would take a form similar to Eq. (28), but its expression would involve two functions a priori different from the functions $F$ and $G$ introduced in Eq. (31). And, extending these coordinates beyond $R=2 M$, one could probably construct a system of Kerr-Schild-like coordinates for $\bar{g}^{\prime}$ too, where the metric resembles (26); but the expression of $\bar{g}^{\prime}$ in these coordinates would not coincide with Eq. (26).

One might then wonder why we chose to evaluate the mean metric corresponding to a finite precision in the measurements of the spatial Kerr-Schild coordinates, and not the Schwarzschild ones. The reason is twofold. First, from a geometrical point of view, the use of Kerr-Schild coordinates is more natural because, as repeatedly stated, these coordinates constitute a single-chart atlas of the space-time manifold describing a Schwarzschild black hole, whereas the Schwarzschild coordinates are only valid outside (or inside) the horizon. As a consequence, practically any discussion of physics around a black hole is made easier by the use of Kerr-Schild coordinates. This relative simplicity will be used in subsequent publications, where the properties of the metric $\bar{g}$ (Eq. (26)) will be further investigated. The other mean metric $\bar{g}^{\prime}$ is interesting too, but its study and comparison with $\bar{g}$ has been knowingly left for a later time.

Order of magnitude and signs of the apparent energydensity, pressures and scalar curvature. Comparison with the de Sitter vacuum. Let us now discuss the main result of this article, namely expressions (37) for the energy-density and pressure associated with the apparent matter and expression (39) for the corresponding scalar curvature of space-time.

All these quantities clearly tend to zero as $R$ tends to infinity. A rough quantitative estimate of the cumulated effect of the coarse-graining is the ratio $\rho$ of the mass-energy of the apparent matter contained in the volume $R>2 M$ to the mass $M$ of the black hole. In order of magnitudes, one finds:

$\rho \sim \frac{1}{M} \int_{2 M}^{+\infty} \frac{a^{2} M^{2}}{R^{6}} R^{2} \mathrm{~d} R$,

so that

$\rho \sim \frac{a^{2}}{M^{2}}$

Thus, $a \sim M / 10$ leads to $\rho \sim 1 / 100$ whereas $a \sim M$ gives $\rho \sim 1$; naturally, this last typical value of $\rho$ for $a \sim M$ should not be taken too seriously because all the calculations presented in this manuscript were made under the assumption $R \gg a$. If $a \sim M$, our evaluation of the mean metric $\bar{g}$ breaks down for $R$ too close to $2 M$ and so does our evaluation of $\rho$. The calculation nevertheless indicates that a coarse-graining $a \sim M$ will probably produce an apparent matter of mass-energy at least comparable to the mass $M$ of the black hole. The effect will probably be even more important if $a>M$ or $a \gg M$. 
Another point deserves further comment. Indeed, the energy density and one of the two pressures of the apparent matter are negative. Imagine now an observer who has access, beyond the coarse-grained metric (26), to a direct evaluation of the mean value $\langle T(\omega)\rangle$ of $T(\omega)$, which vanishes identically. This observer may then interpret Eq. (37) by associating to the "vacuum" a non-vanishing energy density and two pressures, a negative one and a positive one. As already pointed out, this brings to mind the recent observational evidence (Spergel et al. 2003) for a non-vanishing cosmological constant $\Lambda$. Let us now elaborate on this.

The observed cosmological constant is positive. It thus endows the large-scale cosmological vacuum with positive energy-density $\varepsilon_{\Lambda}=\Lambda$ and a (single) negative pressure ${ }^{3} p_{\Lambda}$, which is exactly the opposite of the vacuum energy-density. The cumulated effect of both $\varepsilon_{\Lambda}$ and $p_{\Lambda}$ is best displayed by evaluating two different scalar quantities; the first of these invariants is the associated scalar curvature $\mathcal{R}_{\Lambda}$ of the cosmological vacuum, defined as the scalar curvature of the "empty" de Sitter universe with vacuum stress-energy tensor $\mathcal{T}^{\mu \nu}=$ $\Lambda g^{\mu \nu}$; the second scalar $\tilde{\mathcal{R}}_{\Lambda}$ reflects the "magnitude" of the socalled trace-free Ricci tensor (Penrose \& Rindler 1984) of the same space-time:

$\tilde{\mathcal{R}}_{\Lambda}=8 \pi\left[\left(\mathcal{T}_{\mu \nu}-\frac{1}{4} \mathcal{T} g_{\mu \nu}\right)\left(\mathcal{T}^{\mu \nu}-\frac{1}{4} \mathcal{T} g^{\mu \nu}\right)\right]^{1 / 2}$.

A direct calculation gives (Hawking \& Ellis 1973) $\mathcal{R}_{\Lambda}=-4 \Lambda \times$ $8 \pi$ and $\tilde{\mathcal{R}}_{\Lambda}=0$. In particular, a positive cosmological constant thus induces a negative curvature on space-time, which traces the hyperbolic character of the de Sitter expansion.

Unlike $\varepsilon_{\Lambda}$, the vacuum energy-density of the coarsegrained Schwarzschild space-time is negative. The pressure tensor of this coarse-grained space-time is anisotropic with two eigen-pressures. One of these eigenpressures is positive, but the other one is negative, as $p_{\Lambda}$. The cumulated effect of these vacuum energy-density and pressures is best compared to the effects of a cosmological term by evaluating the same invariants as those just computed for the de Sitter space-time. Contrarily to $\tilde{\mathcal{R}}_{\Lambda}$, the "magnitude" $\tilde{\mathcal{R}}$ of the trace-free Ricci tensor associated with the averaged Scwarzschild space-time is found to be non-vanishing. Indeed, a direct calculation shows that:

$\tilde{\mathcal{R}}=\frac{18 \sqrt{2}}{5} \frac{a^{2} M^{2}}{R^{6}}$.

But the scalar-curvature $\mathcal{R}$ is, as $\mathcal{R}_{\Lambda}$, negative (see Eq. (39)). We think this striking result might indicate that at least part of the cosmological vacuum stress-energy may be due to the large-scale averaging of small-scale structures in the Universe. This claim or hypothesis can surely not be proven with the material presented in this article, but our results indicate that this point deserves a more extended investigation.

In the meantime, it is very tempting to try and extrapolate at least the order of magnitudes indicated by our results to a more general astrophysical or cosmological context. This is the purpose of our next paragraph.

\footnotetext{
3 The pressure tensor is then isotropic.
}

Further comments about the possible astrophysical or cosmological implications of our results. Let us now extrapolate what has hitherto been presented up to cosmological scales. Our reasoning below is only heuristic and we give the result "as is", hoping to provide at least a loose order of magnitude for the gravitational effects of fluctuations in the large-scale Universe.

The results above suggest that the difference between observing a "point-like" object of mass $M$ and an object of mass $M$ spread over a characteristic spatial scale (distance) $a$ can lead to a difference in the observed energy-density which scales as:

$\varepsilon \sim \frac{1}{8 \pi} G \frac{a^{2} M^{2}}{d^{6}}$,

$d$ being the "distance" from the object; here we have abandoned the canonical units and have introduced the gravitational constant $G$ in view of subsequent numerical estimates.

This suggests that the difference between observing a homogeneous object of mass density $\rho$ and spatial size $L$ and a non-homogeneous medium of average density $\rho$, and size $L$ as well, but having fluctuations of order $\delta \rho$ at characteristic spatial scale $a$, leads to a difference in energy-density which might behave like:

$\delta \varepsilon \sim \frac{1}{8 \pi} G \frac{a^{2}\left(\delta \rho L^{3}\right)^{2}}{d^{6}}$

at distance $d$ from the object. So, following this line of reasoning, the relative variation in energy-density, defined as the ratio of $\delta \varepsilon$ by the average mass-energy $\rho c^{2}$ of the object, would behave like

$\frac{\delta \varepsilon}{\rho c^{2}} \sim \frac{1}{8 \pi} \frac{G}{c^{2}} \rho \frac{a^{2}\left(\frac{\delta \rho}{\rho} L^{3}\right)^{2}}{d^{6}}$.

Let us now boldly apply (46) to the universe itself; this might give some indications on how important the averaging of inhomogeneities might be on the cosmological scale. Let $L_{\mathrm{U}}$ be the Hubble-length and suppose that the characteristic spatial scale of the variations is $a=\alpha L_{\mathrm{U}}$. Suppose also that the observation is made at a distance $d=\lambda L_{\mathrm{U}}$. We get

$\frac{\delta \varepsilon}{\rho c^{2}} \sim \frac{1}{8 \pi} \frac{G \rho_{\mathrm{U}} L_{\mathrm{U}}^{2}}{c^{2}} \frac{\alpha^{2}}{\lambda^{6}}\left(\frac{\delta \rho}{\rho}\right)^{2}$

where $\rho_{\mathrm{U}}$ stands for the mean density of the Universe.

Both $\alpha$ and $\delta \rho / \rho$ characterize the fluctuations and $\lambda$ characterizes the distance of observation. On the other hand, $G \rho_{\mathrm{U}} L_{\mathrm{U}}^{2} / c^{2}$ does not depend on the fluctuations themselves or on the distance from which they are observed. In the standard cosmological context, this ratio therefore plays the role of a "fundamental" constant which fixes the order of magnitude of the vacuum stress-energy obtained by averaging a given fluctuation. If one chooses $\rho_{\mathrm{U}}=\rho_{\text {lum }}$, the density of the luminous matter in the universe, one finds, with (Kolb \& Turner 1990) $G=6.7 \times 10^{-8} \mathrm{~cm}^{3} \mathrm{~g}^{-1} \mathrm{~s}^{-2} ; \rho=10^{-29} \mathrm{~g} \mathrm{~cm}^{-3} ; L_{\mathrm{U}}=10^{28} \mathrm{~cm}$ and $c=3 \times 10^{10} \mathrm{~cm} \mathrm{~s}^{-1}$ :

$\frac{G \rho_{\mathrm{U}} L_{\mathrm{U}}^{2}}{c^{2}}=0.07$. 
One can also set $\rho_{\mathrm{U}}$ equal to the critical density $\rho_{\text {crit }}$. The critical density is given (Kolb \& Turner 1990) by

$\rho_{\text {crit }}=\frac{3}{8 \pi} \frac{c^{2}}{G L_{\mathrm{U}}^{2}}$

and one then obtains:

$\frac{G \rho_{\text {crit }} L_{\mathrm{U}}^{2}}{c^{2}}=\frac{3}{8 \pi} \approx 0.12$

in good agreement with Eq. (48), as far as the order of magnitude is concerned. This result practically means that non-linear statistical effects in General Relativity tend to show up precisely at densities around the critical one, which seems quite natural. This indicates that averaging both gravitational fields and energy-densities on a cosmological scale may lead to highly non-trivial and possibly systematic effects whose complete study is however beyond the scope of the present article.

\section{Summary and conclusion}

This article has been devoted to a first application of the averaging formalism for general relativistic gravitational fields presented in Debbasch $(2004,2005)$. We have considered a particular statistical ensemble of space-times which can be physically interpreted as representing a Schwarzschild black hole observed with a finite precision in "spatial" coordinate measurements. The mean gravitational field associated with this ensemble is not a vacuum solution to Einstein's equation. On the contrary, the mean space-time appears as filled with matter; the non-vanishing stress-energy tensor of this apparent matter has been calculated explicitely for points whose "distance" to the black hole is much larger than the retained coarse-graining. The apparent matter can be characterized by an energy density and two distinct pressures. The energy density and one of the pressures are negative, while the other pressure is positive. The overall effect can be traced by the associated apparent scalar curvature of the vacuum regions, which is negative. This effect brings to mind the negative scalar curvature associated with a positive cosmological constant and this point has been discussed thoroughly; in particular, the above similarity not withstanding, there is naturally a difference between the obtained mean space-time and de Sitter space-time; this difference is reflected by the trace-free Ricci tensor, which vanishes for de Sitter space-time and does not vanish for the mean space-time which describes a Schwarzschild black-hole observed with finite precision.

Moreover, extrapolating the conclusions of this article to a broader astrophysical or cosmological context, we have argued that averaging gravitational fields and energy-densities on a cosmological scale might induce some highly non-trivial and possibly systematic effects, endowing for example the cosmological vacuum with a non-vanishing apparent stress-energy density.

Let us finally mention some of the many possible extensions to this work. One should first of all study systematically the coarse-grained metric obtained in this article. What are the geodesics in this gravitational field? Is there an event- or a Cauchy-horizon? And, if the coarse-grained "object" qualifies as a black hole, how is the entropy of this coarse-grained black hole related to the entropy of the unaveraged Schwarzschild black hole?

As already mentioned, the same work should also be undertaken on other statistical ensembles of Schwarzschild black holes, associated with a physically different coarse-graining (for example, a coarse graining in Schwarzschild coordinates and not in Kerr-Schild coordinates); and, naturally, one should also evaluate the effects of finite precision measurements on Kerr black holes.

The very general question one would like to answer is: how does an arbitrary, spatially and temporally fluctuating gravitational field appear after coarse-graining? In particular, is the scalar curvature of the mean space-time always lower than the curvature of the unaveraged space-time? And, more precisely, what about the energy-density and pressures of the apparent matter? These questions will probably be best answered numerically. A first step in this direction may be the study of a collection of randomly placed black holes, which would thus serve as a very crude model of "fluctuating" space-time. We hope to address these questions in subsequent publications. Their importance to astrophysics, cosmology, quantum field theory in curved space-time and quantum gravity can surely not be overestimated.

\section{References}

Buchert, T. 2000, Gen. Rel. Grav., 32, 105

Buchert, T. 2001, Gen. Rel. Grav., 33, 1381

Chandrasekhar, S. 1992, The Mathematical Theory of Black-Holes (Oxford: Oxford University Press)

Debbasch, F. 2004, Eur. Phys. J. B, 37, 257

Debbasch, F. 2005, Eur. Phys. J. B, 43, 143

Futamase, T. 1991, Prog. Theor. Phys., 86, 389

Futamase, T. 1993, Prog. Theor. Phys., 89, 581

Futamase, T. 1996, Phys. Rev. D, 53, 681

Grimmett, G., \& Stirzaker, D. 1994, Probability and Random Processes, 2nd ed. (Oxford: Oxford University Press)

Hawking, S., \& Ellis, G. 1973, The large scale structure of space-time, Cambridge Monographs on Mathematical Physics (Cambridge: Cambridge University Press)

Kasai, M. 1992, Phys. Rev. D, 69, 2330

Kolb, E., \& Turner, M. 1990, The Early Universe, Frontiers in Physics (Redwood City: Addison-Wesley Publishing Company)

Kramer, D., Stephani, H., MacCallum, M., \& Herlt, E. 1980, Exact solutions of Einstein's field equations, Cambridge Monographs in Mathematical Physics (Cambridge: Cambridge University Press)

Landau, L., \& Lifshitz, E. 1975, The Classical Theory of Fields, 4th ed. (Oxford: Pergamon Press)

Nakahara, M. 1990, Geometry, Topology and Physics (Bristol and Philadelphia: Institute of Physics Publishing)

Peebles, P. 1993, Principles of Physical Cosmology, Princeton Series in Physics (Princeton: Princeton University Press)

Penrose, R., \& Rindler, W. 1984, Spinors and space-time (Cambridge: Cambridge University Press)

Spergel, D. N., Verde, L., Peiris, H. V., et al. 2003, ApJS, 148, 175

Wald, R. 1984, General Relativity (Chicago: The University of Chicago Press)

Zalaletdinov, R. 1997, Bull. Astron. Soc. India, 25, 401 